Dyla Tavares de Sá 1

Vera Helena Ferraz de Siqueira 1

Maristela Arantes Marteleto 1

\section{Demanda e clientela multiprofissional: influências e desafios para um Mestrado em Tecnologia Educacional nas Ciências da Saúde}

\author{
Multiprofessional demand and clientele: \\ influences and challenges for a Master's \\ degree course in Educational Technology \\ in the Health Sciences
}

1 Núcleo de Tecnologia Educacional para a Saúde, Centro de Ciências da Saúde, Universidade Federal do Rio de Janeiro. Av. Brigadeiro Trompowski s/no, sala 26, Rio de Janeiro, RJ, 21941-590, Brasil.

\begin{abstract}
This article aims to identify the key characteristics of individuals pursuing a Master's degree in Health Education, discussing relevant issues concerning the objectives and content of their training. The analysis is based on data for Master's applicants selected during the four years since the program on Educational Technology in Health Sciences was created by the Unit of Educational Technology in Health, under the Health Sciences Center at the Federal University of Rio de Janeiro. The study showed that the applicants came from various professions and belonged to both teaching faculty and health care teams. The profile identified by the study has provided the material for discussing key aspects related to the Master's course characteristics and the challenges involved in achieving its social role and meeting the clientele's needs.

Key words Health Education; Graduate Education; Educational Technology; Health Personell; Public Health

Resumo Este artigo tem por objetivo identificar características de indivíduos que procuram uma formação na área da educação em saúde, em nível de pós-graduação. Visa, também, problematizar algumas questões referentes à relação entre essa clientela e os objetivos e conteúdos da proposta do curso. A análise baseia-se em dados sobre a clientela inscrita e selecionada ao longo dos quatro anos de existência do curso de mestrado Tecnologia Educacional nas Ciências da Saúde, do Núcleo de Tecnologia Educacional para a Saúde, Centro de Ciências da Saúde, Universidade Federal do Rio de Janeiro. Entre outros aspectos, encontrou-se que a clientela, em razão da natureza de sua graduação e de seu trabalho, caracteriza-se pela multiprofissionalidade na área da saúde, trabalhando na docência elou na prática assistencial. O perfil encontrado serviu de base para discutir mudanças que vêm ocorrendo na configuração do curso, apresentando desafios para que a proposta cumpra seus papel social e atenda às necessidades da clientela. Palavras-chave Educação em Saúde; Educação de Pós-graduação; Tecnologia Educacional; Pessoal de Saúde; Saúde Pública
\end{abstract}




\section{Introdução}

Os cursos de pós-graduação têm sido objeto de estudos diversos, muitos dos quais dentro do processo de avaliação da CAPES, como em Gazola (1996) e Durham (1996), oferecendo análises importantes para se entenderem tendências, conformação das diferentes áreas e modelos curriculares. Entretanto, identifica-se uma carência de estudos voltados à caracterização de demandas e clientelas dos cursos de pós-graduação no contexto brasileiro. Estudos no gênero são importantes pela contribuição que trazem para se identificarem necessidades concretas de formação, respaldando a relevância da oferta de cursos em determinadas áreas do conhecimento. Contribuem também para reflexões sobre a proposta dos cursos, uma vez que a conformação e identidade dos mesmos tem como um dos atores principais o aluno, com suas especificidades, tais como as decorrentes da natureza de sua formação e trabalho.

É nesse contexto que se insere o presente estudo, no qual se descrevem e discutem as características de indivíduos que procuram o curso de mestrado Tecnologia Educacional nas Ciências da Saúde, do Núcleo de Tecnologia Educacional para a Saúde (NUTES), instituição voltada à elaboração, análise e experimentação de propostas metodológicas de ensino na área de saúde, que atua, desde 1972, como unidade suplementar do Centro de Ciências da Saúde (CCS) da Universidade Federal do Rio de Janeiro (UFRJ). Criado em 1995, esse mestrado teve sua proposta original reformulada em 1998, conforme se discute neste artigo, quando sua denominação foi alterada de Educação em Saúde-Tecnologia Educacional para Tecnologia Educacional nas Ciências da Saúde.

A demanda e clientela são identificadas valendo-se das variáveis dos indivíduos - sexo, idade, tempo e área de formação e natureza do trabalho. Relacionam-se essas características à proposta do programa de mestrado, discutindo-se influências mútuas entre os interesses e necessidades trazidos pelos mestrandos e a configuração do curso. Utilizam-se, neste trabalho, dados do projeto de avaliação do mestrado, ora em andamento. Reconhecem-se as limitações da análise, restrita a um único curso de Tecnologia Educacional nas Ciências da Saúde, criado há apenas quatro anos.

\section{A proposta do mestrado Tecnologia Educacional nas Ciências da Saúde do NUTES}

Desde sua criação, há 25 anos, o NUTES tem concentrado esforços na formação didáticopedagógica de profissionais de saúde, seja em nível de extensão ou de pós-graduação. Até 1983, ofereceu principalmente cursos de curta duração, freqüentados por um total de 6.918 alunos de diferentes estados brasileiros e países da América Latina. A partir de então, o NUTES passou a concentrar seus esforços na oferta de disciplinas de formação didático-pedagógica dos cursos de mestrado do CCS e de curso de especialização em Educação em Saúde, que formou um total de 234 especialistas. O curso de especialização desenvolveu-se ao longo de dez anos (1983-1993), em moldes bastante próximos a um curso de mestrado. Em 1995, dá-se início ao curso de mestrado em Tecnologia Educacional nas Ciências da Saúde, com o objetivo de "formar profissionais qualificados para desempenhar atividades de ensino e pesquisa no campo da educação em saúde, incorporando, de forma crítica, os avanços da tecnologia educacional" (NUTES/UFRJ, 1995).

Ao que se sabe, esse mestrado é o único que adota a tecnologia educacional como eixo, propondo-se a utilizar métodos e teorias que permitam articular a formação didático-pedagógica desenvolvida no curso com a prática profissional dos mestrandos. Está organizado a partir da institucionalização da linha básica de pesquisa Processos e Materiais Educativos nas Ciências da Saúde, que se desdobra em três áreas temáticas articuladas e inter-relacionadas, a saber: a) novas tecnologias da informação e da comunicação na área de educação em saúde, cujos estudos estão centrados nos processos de construção e uso dessas tecnologias; b) avaliação de processos e materiais educativos, voltada para a criação e utilização de modelos qualitativos que permitam a aferição do valor dos mesmos; c) formação da identidade do profissional da saúde, que dá relevo à relação entre a construção da identidade dos profissionais de saúde e suas experiências de vida pessoal e acadêmica. Dessa forma, o mestrado incorpora pressupostos que orientam a produção de conhecimento no NUTES, o primeiro dos quais dá conta de que as tecnologias da informação e da imagem consolidam-se como campo de conhecimento na área da educação nas ciências da saúde, exigindo redefinição de teorias de aprendizagem, da prática pedagógica e dos papéis tradicionalmente desempenhados por professores e alunos no processo ensi- 
no-aprendizagem. O segundo indica a necessidade de uma nova compreensão da prática profissional na saúde e na educação, qual seja, entender a atividade assistencial enquanto prática educativa.

Trata-se de mestrado com perspectiva interdisciplinar, que se consubstancia na articulação intrínseca entre diferentes campos do conhecimento. Desenvolve-se na interseção das áreas de educação, comunicação e saúde, contando para tanto com professores com formação e experiência de trabalho a elas relacionadas. Atualmente, integram o corpo docente permanente professores doutores oriundos das áreas de: educação, psicologia, tecnologia educacional, comunicação/cinema, informática na educação, medicina, saúde pública e sociologia do conhecimento; além disso, a maioria dos profissionais possui larga experiência no campo da educação em saúde.

O curso é aberto a indivíduos das diferentes áreas do conhecimento, portadores de diploma de nível superior. Para explicar o direcionamento que se vem dando ao currículo, segue caracterização da demanda.

\section{Características da demanda}

De um total de 210 candidatos inscritos nos quatro anos, foram selecionados 36 para cursar o mestrado, sendo dez para ingresso em 1995, oito, em 1996, nove, em 1997 e nove, em 1998. Atualmente (1998), conta-se com 31 alunos, tendo havido duas desistências e três defesas de dissertação (cabe aqui esclarecer que o não-reconhecimento do mestrado pela CAPES até dezembro de 1998 causou um retardamento das defesas, prevendo-se que pelo menos 12 trabalhos serão defendidos no decorrer de 1999). Nas quatro seleções estavam disponíveis dez vagas/ano, sem obrigatoriedade do preenchimento de todas. As inscrições para a primeira (1995) e para a terceira turma (1997) caracterizaram-se por uma procura coincidente de 6,4 candidatos por vaga. Em 1996, por causa, provavelmente, de uma divulgação menos abrangente das inscrições, a relação candidato/vaga caiu para 3,8 . As inscrições para a quarta turma apresentaram uma relação candidato/vaga de 4,4, mesmo sendo realizadas num período marcado por greves (setembro de 1997).

O mestrado do NUTES tem atraído candidatos com características variadas - recém-formados, docentes universitários, professores de ensinos fundamental e médio (antigos primeiro e segundo graus), profissionais engajados no ensino não formal e em atividades técnicas, graduados em áreas e subáreas diversas, a grande maioria tendo em comum sua atuação na área da saúde. Esse perfil se reflete também entre os selecionados, conforme os dados apresentados a seguir.

\section{Área de graduação}

Observa-se, nos quatro anos (Tabela 1), a presença de candidatos inscritos com graduação em diversas subáreas relacionadas a seis áreas de conhecimento, caracterizando-se, assim, uma demanda multiprofissional. Nota-se a prevalência de candidatos com graduação na área da saúde, sendo as subáreas medicina e enfermagem as de maior concentração de candidatos inscritos e selecionados. Reforça esse quadro o número de graduados em Serviços Sociais e Psicologia, áreas reconhecidamente afins com a saúde. A distribuição dos selecionados entre as graduações profissionais das diversas áreas e a prevalência da área da saúde fica também evidenciada na Tabela 1 .

Verificou-se que, do total de candidatos inscritos nos quatro anos, 147 possuem algum tipo de especialização lato-sensu. Destes, 127 têm sua especialização em continuidade à própria graduação, sendo 87 na área das ciências da saúde, 32 em ciências humanas e oito em ciências sociais aplicadas. Os demais registram especialização nas áreas de planejamento ambiental (um em 1998), finanças (um em 1998), instrumentos de laboratório (1 em 1998) e educação em saúde (cinco em 1995, dois em 1996, seis em 1997 e quatro em 1998), tendo oito destes últimos cursado essa especialização no NUTES. Dos 36 selecionados, 31 possuem especialização, sendo vinte na área das ciências da saúde, três na área de ciências humanas, dois na área de ciências sociais aplicadas e seis em educação em saúde, sendo quatro com especialização no NUTES.

\section{Tempo de formado}

Pelo exposto na Tabela 2, ocorre um gradativo aumento do tempo de formado dos candidatos inscritos nos quatro anos e uma concentração maior daqueles com nove anos ou mais de sua formatura. A mesma concentração se verifica nos candidatos selecionados, observando-se também que, entre eles, estão representadas todas as outras faixas de tempo de formado.

\section{Atividade profissional}

A grande maioria dos candidatos inscritos e selecionado nesses quatro anos está engajada em 
Tabela 1

Distribuição dos candidatos inscritos e selecionados por áreas e subáreas de graduação e por ano.

\begin{tabular}{|c|c|c|c|c|c|c|c|c|}
\hline \multirow[t]{2}{*}{ Áreas de graduação } & \multicolumn{2}{|c|}{1995} & \multicolumn{2}{|c|}{1996} & \multicolumn{2}{|c|}{1997} & \multicolumn{2}{|c|}{1998} \\
\hline & insc. & selec. & insc. & selec. & insc. & selec. & insc. & selec. \\
\hline \multicolumn{9}{|l|}{ Ciências da saúde } \\
\hline Medicina & 17 & 3 & 4 & 2 & 7 & 1 & 6 & 3 \\
\hline Enfermagem & 12 & 1 & 10 & 3 & 16 & 4 & 11 & 3 \\
\hline Nutrição & 3 & - & 3 & - & 6 & - & 1 & - \\
\hline Odontologia & 4 & 1 & 1 & - & - & - & 1 & - \\
\hline Fonoaudiologia & 2 & - & - & - & 2 & - & 2 & - \\
\hline Educação Física & 1 & - & 3 & - & 5 & 1 & 3 & - \\
\hline Fisioterapia & 1 & - & - & - & 3 & 1 & 4 & 1 \\
\hline Farmácia & 4 & 2 & - & - & - & - & 2 & - \\
\hline Total & 442 & 73 & 21 & 5 & 39 & 7 & 305 & 76 \\
\hline \multicolumn{9}{|l|}{ Ciências humanas } \\
\hline Pedagogia & 4 & - & 2 & - & 3 & - & 2 & - \\
\hline Psicologia & 6 & 1 & 1 & 1 & 6 & 1 & 4 & 1 \\
\hline Total & 10 & 1 & 34 & 1 & 9 & 1 & 6 & 1 \\
\hline \multicolumn{9}{|c|}{ Ciências sociais aplicadas } \\
\hline Serviço Social & 8 & 2 & 9 & 1 & 5 & 1 & 1 & - \\
\hline Comunicação Social & 1 & - & 1 & - & 1 & - & - & - \\
\hline Ciências Sociais & 1 & - & - & - & - & - & - & - \\
\hline Administração & - & - & - & - & 3 & - & - & - \\
\hline Ciências Econômicas & - & - & 1 & - & 2 & - & 1 & - \\
\hline Total & 10 & 2 & 11 & 1 & 11 & 1 & 2 & - \\
\hline \multicolumn{9}{|l|}{ Ciências biológicas } \\
\hline Biologia & 2 & 1 & 3 & 1 & 5 & - & 4 & 1 \\
\hline Total & 2 & 1 & 3 & 1 & 5 & - & 4 & 1 \\
\hline Outras 1 & 1 & - & 1 & - & - & - & 4 & 1 \\
\hline Total geral & 64 & 10 & 38 & 8 & 64 & 9 & 44 & 9 \\
\hline
\end{tabular}

1 Letras, Ciências Exatas, Música, Química Industrial e Desenho Industrial.

Tabela 2

Distribuição dos candidatos inscritos e selecionados por tempo de formado e por ano.

\begin{tabular}{|c|c|c|c|c|c|c|c|c|}
\hline \multirow[t]{2}{*}{ Tempo de formado } & \multicolumn{2}{|c|}{1995} & \multicolumn{2}{|c|}{1996} & \multicolumn{2}{|c|}{1997} & \multicolumn{2}{|c|}{1998} \\
\hline & insc. & selec. & insc. & selec. & insc. & selec. & insc. & selec. \\
\hline 0 a 2 anos & 9 & 1 & 9 & 2 & 7 & - & - & - \\
\hline 3 a 5 anos & 7 & 1 & 3 & 1 & 5 & 2 & 8 & 2 \\
\hline 6 a 8 anos & 10 & 1 & 1 & - & 10 & 1 & 6 & - \\
\hline 9 a 11 anos & 14 & 2 & 7 & 1 & 17 & 2 & 14 & 3 \\
\hline 12 anos ou + & 24 & 5 & 18 & 4 & 25 & 4 & 16 & 4 \\
\hline Total & 64 & 10 & 38 & 8 & 64 & 9 & 44 & 9 \\
\hline
\end{tabular}

* Foram considerados somente anos inteiros de formados após a graduação. 
Distribuição dos candidatos inscritos e selecionados por áreas de atuação profissional e tipo de atividade por ano.

\begin{tabular}{|c|c|c|c|c|c|c|c|c|}
\hline \multirow{2}{*}{$\begin{array}{l}\text { Áreas de atuação } \\
\text { profissional }\end{array}$} & \multicolumn{2}{|c|}{1995} & \multicolumn{2}{|c|}{1996} & \multicolumn{2}{|c|}{1997} & \multicolumn{2}{|c|}{1998} \\
\hline & insc. & selec. & insc. & selec. & insc. & selec. & insc. & selec. \\
\hline \multicolumn{9}{|l|}{ Ciências da saúde } \\
\hline Ensino Universitário1 & 19 & 2 & 11 & 3 & 20 & 5 & 9 & 4 \\
\hline Fundamental e médio & 1 & 1 & 3 & 1 & 6 & 1 & 3 & 2 \\
\hline Não formal & 12 & 3 & 6 & 3 & 10 & 1 & 10 & 2 \\
\hline Atividade técnica & 20 & 2 & 6 & - & 18 & 2 & 15 & 1 \\
\hline Total & 52 & 8 & 26 & 7 & 54 & 9 & 37 & 9 \\
\hline \multicolumn{9}{|l|}{ Ciências humanas } \\
\hline Ensino Universitário & 2 & - & - & - & - & - & - & - \\
\hline Fundamental e médio & - & - & - & - & - & - & 1 & - \\
\hline Não formal & - & - & - & - & - & - & 1 & - \\
\hline Atividade técnica & 1 & - & - & - & - & - & - & - \\
\hline Total & 3 & - & - & - & - & - & 2 & - \\
\hline \multicolumn{9}{|l|}{ Ciências sociais aplicadas } \\
\hline Ensino Universitário & - & - & - & - & - & - & - & - \\
\hline Fundamental e médio & - & - & - & - & - & - & - & - \\
\hline Não formal & - & - & - & - & - & - & - & - \\
\hline Atividade técnica & 1 & - & 3 & - & 2 & - & - & - \\
\hline Total & 1 & - & 3 & - & 2 & - & - & - \\
\hline \multicolumn{9}{|l|}{ Ciências biológicas } \\
\hline Ensino Universitário & 1 & 1 & 2 & - & 2 & - & - & - \\
\hline Fundamental e médio & 1 & - & - & - & 2 & - & 2 & - \\
\hline Não formal & - & - & - & - & - & - & 1 & - \\
\hline Atividade técnica & - & - & - & - & - & - & - & - \\
\hline Total & 2 & 1 & 2 & - & 4 & - & 3 & - \\
\hline \multicolumn{9}{|l|}{ Outras } \\
\hline Ensino Universitário & - & - & - & - & - & - & - & - \\
\hline Fundamental e médio & - & - & 1 & - & - & - & - & - \\
\hline Não formal & - & - & 1 & - & - & - & - & - \\
\hline Atividade técnica & - & - & - & - & - & - & - & - \\
\hline Total & - & - & 2 & - & - & - & - & - \\
\hline Total geral & 58 & 9 & 33 & 7 & 60 & 9 & 42 & 9 \\
\hline Nenhuma & 6 & 1 & 5 & 1 & 4 & - & 2 & - \\
\hline
\end{tabular}

1 Em torno de 60\% dos docentes universitários da área da saúde exercem também atividade técnica (assistência) mas foram contados uma única vez no tipo de atividade indicado por eles como de maior peso (ensino).

alguma atividade nos ensinos universitário, fundamental e médio, no ensino não formal ou em atividade técnica, notadamente na área da saúde, conforme a Tabela 3, que foi organizada com categorias exclusivas. Por isso, é importante lembrar que os profissionais docentes da área da saúde (médicos, enfermeiros, psicólogos) estão, em geral, também envolvidos em atividades técnicas (assistência). Pode-se observar que, nos dois primeiros anos, o número de professores universitários selecionados foi igual ao de profissionais atuantes no ensino não formal. Os números mudam em 1997, com uma aprovação bem maior de docentes universitários, que é mantida em 1998. Entre os selecionados, estão representados, também, professores de ensinos fundamental e médio, bem como profissionais engajados em atividades técnicas. 


\section{Tipo de empregador}

A grande procura do mestrado por parte de servidores de instituições públicas (Tabela 4) pode ser explicada pelo fato de os profissionais engajados na área da saúde estarem concentrados basicamente no serviço público no Brasil. Dentre os 36 selecionados, dois não trabalham, cinco pertencem à empresa privada $\mathrm{e}$ os demais 29 são provenientes de serviço público e, na sua grande maioria, das seguintes instituições: Universidade Federal do Rio de Janeiro - UFRJ; Universidade Federal Fluminense - UFF; Fundação Oswaldo Cruz - FIOCRUZ; Universidade do Estado do Rio de Janeiro - UERJ; Universidade do Rio de Janeiro UNI-RIO.

\section{Faixa etária}

Há uma concentração maior dos candidatos inscritos em 1995 nas faixas compreendidas entre 26 e 37 anos, com um deslocamento des- sa concentração, em 1996, para as faixas entre 32 e 43 anos, repetido em 1997 e 1998 (Tabela 5). Observa-se, também, uma diminuição, ou desaparecimento, de um ano para outro até 1997, de candidatos nas faixas etárias de 20 a 25 e de cinqüenta anos ou mais, que voltam a aparecer, no entanto, em 1998. Entre os selecionados, considerando-se os quatro anos de existência do mestrado, todas as faixas etárias estão representadas, com maior predominância, no entanto, nas faixas entre 32 e 49 anos.

\section{Sexo}

A Figura 1 apresenta o panorama geral dos candidatos, em cada um desses três anos, confirmando, tanto entre os inscritos, quanto entre os selecionados, a predominância histórica do sexo feminino em programas de graduação ou de pós-graduação relacionados à área da educação. Essa constatação é também coerente com a tendência de crescimento da participação da mão-de-obra feminina na área da

Tabela 4

Distribuição dos candidatos inscritos e selecionados por tipo de empregador e por ano.

\begin{tabular}{|c|c|c|c|c|c|c|c|c|}
\hline & \multicolumn{2}{|c|}{1995} & \multicolumn{2}{|c|}{1996} & \multicolumn{2}{|c|}{1997} & \multicolumn{2}{|c|}{1998} \\
\hline & insc. & selec. & insc. & selec. & insc. & selec. & insc. & selec \\
\hline Público (UFRJ) & 17 & 2 & 10 & 3 & 14 & 3 & 11 & 1 \\
\hline Público (outros) & 26 & 5 & 15 & 3 & 30 & 4 & 24 & 5 \\
\hline Privado & 10 & 1 & 7 & - & 13 & 1 & 7 & 3 \\
\hline Estagiários/bolsistas & 5 & 1 & 1 & 1 & 3 & 1 & 1 & - \\
\hline Não trabalham & 6 & 1 & 5 & 1 & 4 & - & 1 & - \\
\hline Total & 64 & 10 & 38 & 8 & 64 & 9 & 44 & 9 \\
\hline
\end{tabular}

Tabela 5

Distribuição dos candidatos inscritos e selecionados por faixa etária e por ano.

\begin{tabular}{|c|c|c|c|c|c|c|c|c|}
\hline \multirow[t]{2}{*}{ Faixa etária } & \multicolumn{2}{|c|}{1995} & \multicolumn{2}{|c|}{1996} & \multicolumn{2}{|c|}{1997} & \multicolumn{2}{|c|}{1998} \\
\hline & insc. & selec. & insc. & selec. & insc. & selec. & insc. & selec. \\
\hline 20 a 25 & 6 & 1 & 4 & 1 & 2 & - & 1 & - \\
\hline 26 a 31 & 16 & 2 & 3 & 1 & 12 & 2 & 8 & 2 \\
\hline 32 а 37 & 20 & 3 & 15 & 3 & 23 & 5 & 13 & 2 \\
\hline 38 a 43 & 10 & 1 & 12 & 3 & 18 & 1 & 11 & 1 \\
\hline 44 a 49 & 9 & 3 & 4 & - & 9 & 1 & 7 & 2 \\
\hline 50 ou + & 3 & - & - & - & - & - & 4 & 2 \\
\hline Total & 64 & 10 & 38 & 8 & 64 & 9 & 44 & 9 \\
\hline
\end{tabular}




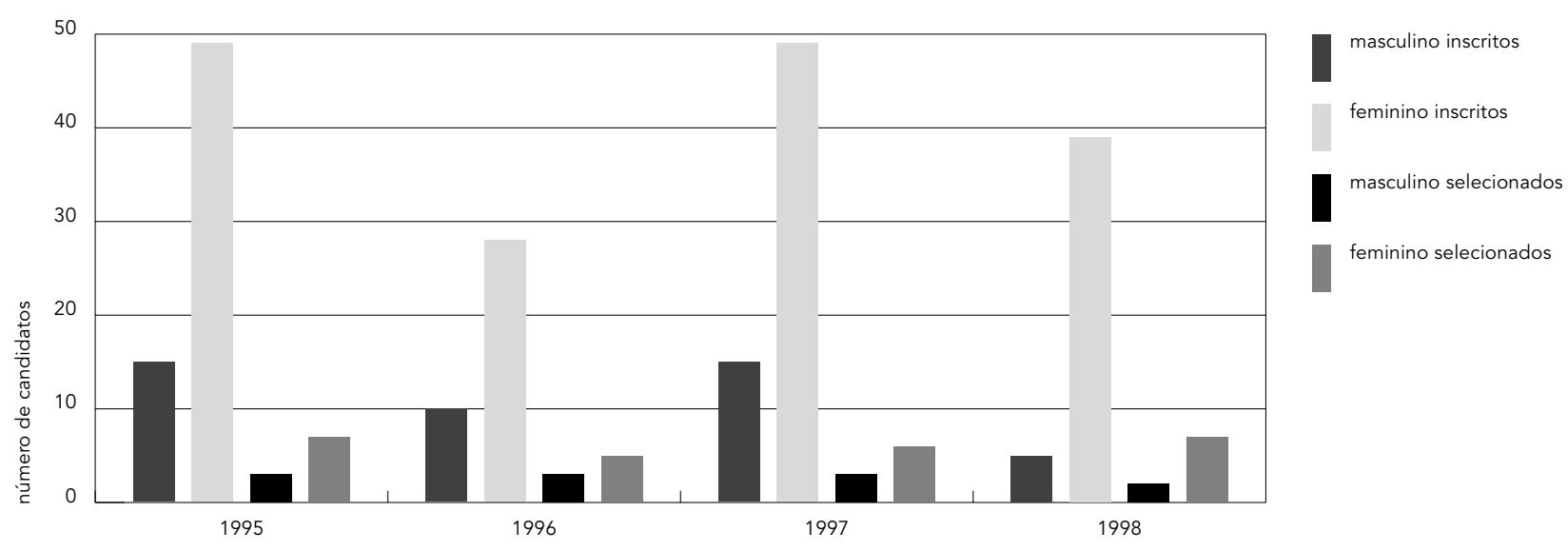

saúde, apontada por análise da força de trabalho em saúde (Machado, 1987).

Os dados apresentados até aqui especificam características da demanda e da clientela que têm se apresentado para o mestrado nesses últimos quatro anos. Embora essas características ainda possam se modificar ao longo dos próximos anos, hoje há uma base de dados que aponta a multiprofissionalidade na área da saúde como principal identificador da demanda da pós-graduação Tecnologia Educacional nas Ciências da Saúde do NUTES.

\section{A multiprofissionalidade na concretização da proposta}

A multiprofissionalidade evidenciada vem, sem dúvida, exercendo influências e colocando desafios à proposta do curso. Entendemos que o currículo atual, após mudanças introduzidas em 1998, proporciona um melhor atendimento à diversidade evidenciada na demanda e na clientela. Menciona-se, em primeiro lugar, a maior flexibilidade imprimida ao programa, com a diminuição de seis para apenas quatro disciplinas obrigatórias: Tecnologia Educacional, Educação em Saúde, Metodologia da Pesquisa e Seminário de Tese. Essa programação, ao mesmo tempo que preserva um núcleo comum de conhecimento, o que se considera imprescindível para manter uma unidade dentro da diversidade, conjuga os objetivos sociais, que expressam a responsabilidade com o aten- dimento à saúde da população, com os objetivos individuais trazidos pelos mestrandos, como recomenda Boavida (1998) ao se referir aos múltiplos condicionantes e a inflexões particulares que caracterizam o processo educativo. Nesse novo plano, os alunos, desde o primeiro semestre, já se engajam obrigatoriamente nas linhas de pesquisa desenvolvidas nos laboratórios do NUTES, dentro de suas respectivas áreas de interesse. Outro aspecto na configuração atual do curso refere-se à exigência de apresentação de um projeto por ocasião da seleção, o que já se constitui numa expressão das necessidades e interesses desses profissionais, permitindo maior integração das atividades curriculares com as realidades trazidas. É assim que o objetivo do projeto do mestrado do NUTES é explicitado atualmente: "Desenvolver, de modo articulado com a estrutura curricular que se oferece, a capacitação dos alunos nas atividades de pesquisa e desenvolvimento de tecnologias educacionais, no âmbito dos projetos dos docentes, criando condições para consolidar e ampliar o interesse pela pesquisa, pela docência em nível superior e pelas ações educativas nos serviços de saúde" (UFRJ, 1998).

Lembra-se que, conforme indicam os dados, grande parte do alunado está ligada à prática assistencial, cuja dimensão educativa tem sua especificidade, não se igualando à prática docente no ensino formal, objeto tradicional da construção do conhecimento pedagógico. Esse fato coloca desafios na consolidação de saberes teórico-metodológicos que possam 
contemplar as peculiaridades das relações diretas que se estabelecem entre profissionais de nível superior e população. Trata-se de uma relação educativa permeada não apenas por diferenças de classe e culturais, mas que, ao se voltar à problemática de saúde/doença, remete a questões de ordem individual, com uma carga acentuada de subjetividade. A constatação de que ainda é escassa a produção de conhecimento sobre essa relação pedagógica aponta para a responsabilidade do programa na realização de estudos que contribuam para suprir tal lacuna, sobretudo focalizando a realidade brasileira.

Os dados apresentados evidenciam que a multiprofissionalidade dos alunos se assemelha à constituição das equipes de saúde, o que permite, pelo estímulo às situações coletivas de atividades curriculares, a troca de saberes e reflexões importantes para o melhor entendimento da assistência à saúde e para o reconhecimento da necessidade de superar as limitações de uma formação em nível de graduação que, de forma geral, não proporciona experiências que integrem o corpo discente das diferentes áreas da saúde. Vale ressaltar também que as discussões dos objetos de estudo são enriquecidas pelos olhares oriundos de campos disciplinares distintos. Ressalta-se que essas discussões mostram-se mais conseqüentes e legítimas, na medida em que se considera que a grande maioria possui, como anteriormente evidenciado, pelo menos nove anos de experiência profissional, trazendo, pois, inquietações e necessidades que emergem de seu cotidiano. A análise da demanda sugere, ainda, com base na identificação do tipo de atividade e das instituições de origem dos candidatos, que muitos já vêm participando de propostas comprometidas com a dimensão educativa, reconhecida como inerente não só ao processo de formação, como também à prática assistencial. Por isso, buscam uma fundamentação que lhes proporcione condições para o amadurecimento dessas ações, cuja complexidade demanda uma perspectiva interdisciplinar.

Essas considerações se apóiam na fala de candidatos e mestrandos em diferentes momentos da seleção e também durante o curso, ao esclarecerem por que procuram esse mestrado:

"Por ser uma área fundamental, básica, para o desempenho de minha função e de todo Docente e Médico, porém ausente em minha (nossa) formação. Lamento que não seja oferecida como disciplina curricular [referindo-se à graduação], pois o Ato Médico não passa de um processo educativo" (Médico, docente da Medicina).
"Por querer sair da especificidade do olhar da enfermagem para a multiplicidade de vários profissionais de saúde ligados com a Educação" (Enfermeira, docente da Enfermagem).

"Porque abria a perspectiva de uma crítica, de reflexão/elaboração teórica, a fim de contribuir para maior clareza acerca dos processos educativos envolvidos na minha própria área. Perspectiva esta não apresentada em minha área de formação" (Farmacêutico).

“ $O$ 'espírito' de construção coletiva do conhecimento concomitante à avaliação do processo tem sido fundamental nessa vivência, pois, 'ilumina' as discussões de grupo, as aulas, enfim, as práticas de ensino do mestrado, promovendo: contribuições de todos; verbalização dos insights'; identificação das contradições e conflitos de nossa prática; (...); consolidação de ações mais planejadas e embasadas em nossas instituições de origem; avaliação e recondução desses novos 'caminhos'... tudo isso se constitui em vivência conscientizada criticamente" (Médica, docente da Medicina e Enfermagem).

Uma reflexão que permeia nossa tentativa de amadurecimento da proposta refere-se à natureza dos trabalhos de dissertação. O perfil da clientela, articulado com a proposta curricular, tem proporcionado investigações que estabelecem novos e mais abrangentes olhares para a tecnologia educacional, renovando e ampliando seu conceito. Como exemplos de dissertações defendidas e a defender proximamente, citam-se estudos que focalizam as relações imagem/corpo/saúde, em diferentes formas de expressão, como vídeos e livros; avançam no significado da dimensão educativa da assistência à saúde; tentam romper com a dicotomia razão/emoção na formação e prática do profissional de saúde, evidenciando as subjetividades na formação de sua identidade; renovam os conceitos de educação presencial e a distância, valorizando modelos interativos no uso da informática, com apoio de abordagens construtivistas. O desenvolvimento de outros formatos distintos das tradicionais dissertações - que incluam, por exemplo, a elaboração de vídeos e textos impressos - parece coerente com a proposta do mestrado e tem sido incentivado, tendo em vista não só a natureza da tecnologia educacional, como as necessidades da prática assistencial no que concerne a procedimentos e materiais mediadores das relações pedagógicas. 


\section{Conclusões}

Entende-se que o fato de o curso trazer como eixo a relação educação-saúde-tecnologia e não uma área específica dentro da saúde já imprime um certo direcionamento à demanda. A multiprofissionalidade em áreas da saúde, representada nos inscritos e selecionados, constitui-se em especificidade do curso, cumprindo duplo papel: ao mesmo tempo que reforça a proposta interdisciplinar, que antecede à concretização da demanda, pressupõe-na e coloca exigências valendo-se de expectativas e experiências de trabalho. Deve-se lembrar que essa dimensão é comum a alguns outros programas de mestrado, inclusive da área de saúde, como os de saúde coletiva. Em relação à demanda, os contornos do programa do mestrado de que trata o presente artigo ganham ainda maior definição quando se identifica a dimensão educativa da saúde como foco de interesse e estudo resultante do universo do trabalho e das preocupações da clientela.

A perspectiva de interdisciplinaridade, proposta do curso reforçada pelo perfil e expectativas da clientela, vai ao encontro de desafios colocados pelas transformações que ocorrem no campo do conhecimento. Primeiramente, exige dos docentes a identificação/adoção de um paradigma que considere a perspectiva da totalidade do conhecimento (Kosik, 1989). Além disso, a seleção e abordagem dos conteúdos trabalhados em cada disciplina deve articular minimamente as três áreas - educação, comunicação e saúde -, configurando, assim, um conhecimento que, sem perder de vista a especificidade da área, 'salte' os muros disciplinares. A incidência dos campos de atuação da clientela do curso (medicina, enfermagem, serviço social etc.), que se dá diretamente sobre a prática social, reforça a desejada e difícil relação dialética entre teoria e prática.

Embora acredite-se na coerência do trabalho, e seja patente o entusiasmo dos que atuam no programa - docentes e mestrandos -, considera-se essencial para a realimentação da proposta que se proceda ao acompanhamento dos ex-alunos, tendo em vista identificar como e/ou até que ponto o educativo e a construção de conhecimentos de educação em saú$\mathrm{de} /$ tecnologia educacional permeiam as suas práticas.

\section{Referências}

BOAVIDA, J., 1998. Educação: Objectivo e subjectivo. Para uma teoria do itinerário educativo. Porto: Porto Editora.

DURHAM, E., 1996. Subsídios para discussão da avaliação do ensino superior. Boletim Informativo da CAPES, 4.

GAZOLA, A. L., 1996. Evolução das formas de organização da pós-graduação brasileira. Boletim Informativo da CAPES, 4.

KOSIK, K., 1989. Dialética do Concreto. Rio de Janeiro: Editora Paz e Terra.

MACHADO, M. H., 1987. A participação da mulher na força de trabalho em saúde no Brasil - 1970-1980. In: Textos de Apoio - Planejamento I: Recursos Humanos em Saúde. pp. 51-60, Rio de Janeiro: ENSP.

NUTES/UFRJ (Núcleo de Tecnologia Educacional para a Saúde/Universidade Federal do Rio de Janeiro), 1995. Mestrado em Tecnologia Educacional nas Ciências da Saúde - Proposta de curso, Rio de Janeiro: NUTES/UFRJ (mimeo.).

NUTES/UFRJ (Núcleo de Tecnologia Educacional para a Saúde/Universidade Federal do Rio de Janeiro), 1998. Mestrado em Tecnologia Educacional nas Ciências da Saúde - Proposta de curso, Rio de Janeiro: NUTES/UFRJ (mimeo.). 